hep-th/9907183, IASSNS-HEP-99/70

CINVESTAV-FIS-99/33

\title{
D-branes on Group Manifolds and Deformation Quantization
}

\author{
Hugo García-Compeán ${ }^{a, b]}$ and Jerzy F. Plebańskib \\ ${ }^{a}$ School of Natural Sciences \\ Institute for Advanced Study \\ Olden Lane, Princeton, NJ 08540, USA \\ ${ }^{b}$ Departamento de Física \\ Centro de Investigación y de Estudios Avanzados del IPN \\ Apdo. Postal 14-740, 07000, México D.F., México
}

\begin{abstract}
Recently M. Kontsevich found a combinatorial formula defining a star-product of deformation quantization for any Poisson manifold. Kontsevich's formula has been reinterpreted physically as quantum correlation functions of a topological sigma model for open strings as well as in the context of D-branes in flat backgrounds with a Neveu-Schwarz Bfield. Here the corresponding Kontsevich's formula for the dual of a Lie algebra is derived in terms of the formalism of D-branes on group manifolds. In particular we show that that formula is encoded at the two-point correlation functions of the Wess-Zumino-Witten effective theory with Dirichlet boundary conditions. The $B$-field entering in the formalism plays an important role in this derivation.
\end{abstract}

July, 1999

\footnotetext{
1 E-mail: compean@fis.cinvestav.mx

2 E-mail: pleban@fis.cinvestav.mx
} 


\section{Introduction}

D-branes are extremely interesting objects which were originally applied to realize string dualities and to probe substringly distances (for a review, see Ref. [1]). One of the most exciting aspects of D-brane physics is the realization and explanation of several mathematical constructions in the language of physical processes of branes. For instance, the ADHM construction of Yang-Mills instantons founds a nice realization in terms of D-brane configurations (for a review of this subject, see Ref. [2]).

Integrability present in the formulation of the string theory on group manifolds, originally studied in [3], provided an excellent guide in the search of a realistic string theory. The study of D-branes on group manifolds was first considered by Klimčik and Ševera in [4] and further developed in [5,6,7] from the point of view of boundary states theory. Some results of Ref. [4] will be of particular usefulness for the purposes of this paper and they will be reviewed at the section 3. Of special interest is the result of [4] that a WZW Lagrangian can be well defined for open strings propagating in a group manifold. This effective Lagrangian can be obtained from the pairing between the relative homology chain and the relative cohomology chain, i.e. $H_{*}\left(\mathbf{G} / \mathbf{H}_{m}, \mathbb{Z}\right) \otimes H^{*}\left(\mathbf{G} / \mathbf{H}_{m}, \mathbb{Z}\right) \rightarrow \mathbb{Z}$ where the integer-valued relative homology and cohomology ensures a well defined Feynman path integral. Here $\mathbf{G}$ is the group manifold and $\mathbf{H}_{m}$ is a group submanifold of $\mathbf{G}$. Within this context the D-branes of various dimensionalities are identified with the submanifolds $\mathbf{H}$ and the open world-sheet $W$ generated $b^{6} y$ the propagation of the open string in $\mathbf{G}$ can be identified with the relative homology cycles of the corresponding dimension. More recently, it has been shown that D-branes identified with the submanifolds $\mathbf{H}_{m}$ correspond to the conjugacy classes of the relevant Lie group G. Thus open strings end points are permitted to be fixed in such a classes and stretched between pairs of them [8] (for a very nice recent review on the subject, see Ref. [9]).

On the other hand very recently a renewed deal of excitation has been taken place in deformation quantization theory [10], since the Kontsevich's seminal paper [11]. In this paper Kontsevich proved by construction the existence of a star-product for any finite dimensional Poisson manifold. His construction is based on his more general statement known as the "formality conjecture". The existence of such a star-product determines the existence of a deformation quantization for any Poisson manifold. Kontsevich's proof was strongly motivated by some perturbative issues of string theory and topological gravity in two-dimensions, such as, matrix models, the triangulation of the moduli space of Riemann surfaces and mirror symmetry [12]. 
Kontsevich's star product can also be defined for the dual of a Lie algebra $\mathcal{G}^{*}$. The structure of $\mathcal{G}^{*}$ as vector space it is known to be isomorphic to $\mathbb{R}^{n}$, being $n$ the number of generators of the Lie algebra $\mathcal{G}$. $\mathcal{G}^{*}$ with the Kirillov-Poisson structure is a Poisson manifold and the Kontsevich's star-product can be defined [11]. It is also well known that other star-products can be defined on $\mathcal{G}^{*}$ for any Lie algebra $\mathcal{G}$. The first of them was obtained by Gutt [13] by studying the Hochschild cohomology of Lie algebras. There it was used not the Kirillov-Poisson structure on $\mathcal{G}^{*}$ but only the symplectic structure on the cotangent bundle to the relevant Lie group (for a recent progress on this subject, see [14]). Gutt's star-product is naturally connected to the termed Campbell-Baker-Hausdorff $(\mathrm{CBH})$ quantization through suitable deformations of the universal enveloping algebras as we shall review in Section 2 following Refs. [15, 16].

The relation of Kontsevich's quantization to the CBH quantization, was amply discussed in [15] (see also [16]). It was shown in these references that both the Kontsevich's and universal enveloping algebra quantization (also called $\mathcal{U}$-quantization), are obtained from the exponentiation of the Kirillov-Poisson structure on $\mathcal{G}^{*}$, through the $\mathrm{CBH}$-formula (for a review about the CBH-formula for the discrete and continuos cases, see [17]). Moreover both quantizations are exactly the same when Kontsevich's formula is restricted to consider only symmetric-admissible graphs (with the loop-graphs being neglected [15]). In Ref. [18], such equivalence is further investigated from the point of view of the formality conjecture. Finally the study of Lie bi-algebras and their quantization was initialized by Drinfeld. He conjectured that every Poisson-Lie group has a canonical quantization. The proof of this conjecture was given recently by Etingof and Kazhdan [19]. Recently a new proof of the formality conjecture was given by Tamarkin [20] involving operads and motives. The implications of this new proof in the deformation quantization theory was more recently given by Kontsevich [21].

Afterwards a path integral interpretation of the Kontsevich's star-product was worked out very recently by Cattaneo and Felder [22]. In this paper Kontsevich's formula is extracted from the three-point correlation functions on the disc from the perturbation theory of a bosonic topological open string theory on the target space provided with the relevant Poisson structure. Formality conjecture issues were also described there in field theoretic terms. Further developments in this context were worked out at [23]. The study of noncommutative gauge theories on Poisson manifolds was recently discussed in [24].

Among various applications of D-branes of particular interest, for the purposes of this paper, is that of the D-brane realization of the Kontsevich's star-product [11]. Kontsevich's 
star product can be also derived from the correlation functions of open string theory in for flat backgrounds, but with non-trivial constant $B$-field [25]. In this paper we pursue a similar derivation of the Kontsevich's quantization formula for the dual of a Lie algebra in field theoretic terms. In particular we show that it can be obtained from the Wess-ZuminoWitten theory for open strings with Dirichlet boundary conditions. To be more precise we will show that that Kontsevich star product is encoded in the effective field theory on the D-brane in group manifolds described in [4].

One of the main lessons of the stringly [22] and D-brane [25] descriptions of Kontsevich's formula in that of the deformation quantization for any Poisson manifold requires necessarily of string theory. The deformation parameter of this quantization is precisely the string scale $\alpha^{\prime}$ (or the string coupling constant) which in the limit $\alpha^{\prime} \rightarrow 0$ it reproduces the field theory limit but in this limit the deformation quantization does not exist. The deformation arising precisely when $\alpha^{\prime} \neq 0$ is an indication that deformation quantization is an stringly phenomenon. Actually it was already suspected since the origin of the formality conjecture where several mathematical ingredients of string theory were present.

Deformation quantization also arises in the description of the behavior of D-branes in type IIA and IIB superstring theory on tori and in a background $B$-field [26]. In particular, the $\mathrm{M}$ (atrix)-theory compactifications on tori have been seen described through a YangMills gauge theory (with sixteen supercharges) on the noncommutative dual tori (for a recent review, see [27] and for some recent progress, see [28,29]). Fedosov deformation quantization can be also incorporated into this context following the lines of [30].

We start in Section 2 by reviewing some basic features of the relevant star-products of the dual Lie algebra $\mathcal{G}^{*}$, we will follow mainly the work of Kathotia [15]. In Section 3 we describe some necessary facts about the theory of D-branes on group manifolds [4]. In Section 4 we discuss the derivation of the Kontsevich's formula for $\mathcal{G}^{*}$ from the theory of D-branes on group manifolds. Finally, Section 5 contains our concluding remarks.

\section{Overview of the Various Star-Products in the Dual of a Lie Algebra}

In this section we shall give an overview of the star-products on the dual Lie algebra $\mathcal{G}^{*}$, in particular of the Kontsevich's star product and its relation with the various starproducts arising in the literature. Our aim is not to provide an extensive review of such 
constructions, but to briefly recall the relevant structure of these quantizations and provide the notation, which we will need in the following sections. For a more complete treatment see Refs. [15, 16,18]. We will follow mainly the notation of Ref. [15].

\subsection{Linear Poisson Structure. $\mathcal{U}$-quantization}

Let us first briefly review the linear Poisson structures. For definiteness we are going to consider the deformation quantization of the dual Lie algebra $\mathcal{G}^{*}$ to the Lie algebra $\mathcal{G}$ associated to a compact and simple Lie group $\mathbf{G} \cdot \mathcal{G}^{*}$ is provided with the canonical KirillovPoisson structure given by the structure constants of $\mathcal{G}, f_{k}^{i j}$ in the way $\left[X^{i}, X^{j}\right]=f_{k}^{i j} X^{k}$, for any pair of elements $X^{i}$ and $X^{j}$ of a basis of $\mathcal{G}$. The Kirillov-Poisson structure on $\mathcal{G}^{*}$ is given by $\alpha=\frac{1}{2} f_{k}^{i j} X^{k} \partial_{i} \wedge \partial_{j}$. Here the elements $X^{i}$ can be seen also as the local basis on $\mathcal{G}^{*}$. Now in order to induce a star-product on the space of smooth functions $C^{\infty}\left(\mathcal{G}^{*}\right)$ on $\mathcal{G}^{*}$, one has to consider a suitable deformation of the universal enveloping algebra $\mathcal{U}\left(\mathcal{G}_{\hbar}\right)$ and the deformation of the symmetric tensor algebra $\mathcal{S}(\mathcal{G})[[\hbar]]$ of $\mathcal{G}$ where $\hbar$ is the deformation parameter. $\mathcal{S}(\mathcal{G})[[\hbar]]$ can be identified with the space of polynomial functions on $\mathcal{G}$ taking values in the infinite formal series in $\hbar$. Thus if $\sigma$ is the algebra isomorphism (given by the symmetrization of monomials through the Poincaré-Birkhoff-Witt theorem) between $\mathcal{U}\left(\mathcal{G}_{\hbar}\right)$ and $\mathcal{S}(\mathcal{G})[[\hbar]]$, it can be induced a star-product on $C^{\infty}\left(\mathcal{G}^{*}\right)[[\hbar]]$ in the form $P \star Q=\sigma^{-1}(\sigma(P) \circ \sigma(Q))$, where $P, Q$ are two elements on $C^{\infty}\left(\mathcal{G}^{*}\right)[[\hbar]]$ and $\circ$ is an associative and noncommutative product in the universal enveloping algebra $\mathcal{U}$ B. Linear Poisson structure of $\mathcal{G}^{*}$ leads naturally to two equivalent quantizations, the $\mathcal{U}$-quantization and the $\mathrm{CBH}$ quantization. For the purposes of this paper is enough to describe more in detail, the CBH-quantization. The star product defined as above is equivalent to the Gutt's star-product defined on the cotangent bundle to the Lie group G [13]. Of course they coincide with the star-product in the direction of the Kirillov-Poisson $\alpha$, that is, $P \star Q=\frac{1}{2} f_{k}^{i j} X^{k} \overleftarrow{\partial}_{i} P \cdot \vec{\partial}_{j} Q$. Thus the star-product on $\mathcal{G}^{*}$ can generated through the $\mathrm{CBH}$-formula. This is the subject of the next subsection.

3 In the above procedure it was used the fact that $C^{\infty}\left(\mathcal{G}^{*}\right)[[\hbar]]$ and $\mathcal{S}(\mathcal{G})[[\hbar]]$ are canonically isomorphic. 


\subsection{Campbell-Baker-Hausdorff Quantization}

The Campbell-Baker-Hausdorff quantization is based in the $\mathrm{CBH}$-formula for the group multiplication. The product of two group elements of the form $\exp (X)$ and $\exp (Y)$ with $X, Y$ being elements of the Lie algebra $\mathcal{G}$ is given by

$$
\exp (X) \cdot \exp (Y)=\exp (H(X, Y))
$$

where $H(X, Y)$ is a series of the form

$$
\left.H(X, Y)=X+Y+\frac{1}{2}[X, Y]+\frac{1}{12}([X,[X, Y]]+[X, Y], Y]\right)+\ldots
$$

with $[X, Y]$ is the Lie bracket in $\mathcal{G}$.

Now we consider a basis of $\mathcal{G}$ given by $\left\{X^{i}\right\}$ with $i=1, \ldots, d$. By using the KirillovPoisson structure, the generators of $\mathcal{G}$ are also seen as coordinates in $\mathcal{G}^{*}$. This permits to establish, for any small real number $t$, the correspondence between the functions $\exp (t X)$ on $\mathcal{G}^{*}$ and the group elements of the form $\exp (t X)$ with $X \in \mathcal{G}$

$$
\text { Functions on } \mathcal{G}^{*} \Longleftrightarrow \text { Group elements } \exp (t X) \text { with } X \in \mathcal{G} \text {. }
$$

Pulling-back the group multiplication to the functions via this correspondence leads to the CBH-quantization. This correspondence will be of prime importance below in the construction of the star-product through the D-brane technology in section 4. Meanwhile we will describe this correspondence with certain detail. Under this correspondence the CBH-formula induces a bi-differential operator $\hat{\mathcal{D}}: C^{\infty}\left(\mathcal{G}^{*}\right) \otimes C^{\infty}\left(\mathcal{G}^{*}\right) \rightarrow C^{\infty}\left(\mathcal{G}^{*}\right)$. The procedure to construct this operator is by constructing first its symbol $D$ and then finding out its corresponding operator. Consider first the relation

$$
\exp (H(X, Y)-X-Y)=\exp \left(\frac{1}{2}[X, Y]+\frac{1}{12}([X,[X, Y]]+[[X, Y], Y])+\ldots\right)
$$

coming from the group multiplication of the elements $\exp (X)$ and $\exp (Y)$. Substituting $X=s_{i} X^{i}$ and $Y=t_{j} X^{j}$ in the above formula, its rhs provides a definition of the symbol $D$ of the operator $\hat{\mathcal{D}}$ given by

$$
D \equiv \exp \left(\frac{1}{2} X^{k} f_{k}^{i j} s_{i} t_{j}+\frac{1}{12}\left(X^{k} f_{k}^{i m} f_{m}^{l j} s_{i} s_{l} t_{j}+X^{k} f_{k}^{m l} f_{m}^{i j} s_{i} t_{j} t_{l}\right)+\ldots\right)
$$


where $s_{i}$ and $t_{i} i, j=1, \ldots, d$ are real valued commuting variables.

This formula tell us that the symbol $D$ depends linearly on the variables $X^{k}$ with $X^{k}$ being the local coordinates on $\mathcal{G}^{*}$ which are commuting variables too. Thus in the definition of the symbol given by Eq. (2.5) there is no noncommuting variables which leads to some ambiguities. The operator $\hat{\mathcal{D}}$ by itself is determined as usual by the substitution of $s_{i} \leftrightarrow \overleftarrow{\partial}_{i}$ and $t_{j} \leftrightarrow \vec{\partial}_{j}$. It is given by

$$
\hat{\mathcal{D}}=\exp \left(\frac{1}{2} X^{k} f_{k}^{i j} \overleftarrow{\partial}_{i} \cdot \vec{\partial}_{j}+\frac{1}{12}\left(X^{k} f_{k}^{i m} f_{m}^{l j} \overleftarrow{\partial}_{i} \overleftarrow{\partial}_{l} \vec{\partial}_{j}+X^{k} f_{k}^{m l} f_{m}^{i j} \overleftarrow{\partial}_{i} \vec{\partial}_{j} \vec{\partial}_{l}\right)+\ldots\right)
$$

Thus $\hat{\mathcal{D}}$ determines the associative star-product on $C^{\infty}\left(\mathcal{G}^{*}\right)$ given by

$$
F \star G \equiv \hat{\mathcal{D}}(F, G)
$$

for the functions $F=\exp (s X)$ and $G=\exp (t Y)$ on $\mathcal{G}^{*}$. This product determines a deformation of the point-wise multiplication in the direction of the Poisson bracket $\alpha$.

The associativity of $\star$ is induced from the associativity of the group multiplication via the pull-back of such a property from the CBH-formula. Written explicitly Eq. (2.7) looks like

$$
\exp \left(s_{i} X^{i}\right) \star \exp \left(t_{j} X^{j}\right)=\hat{\mathcal{D}}\left(\mathbf{X}, \mathbf{f},\left(\overleftarrow{\partial_{1}}, \ldots, \overleftarrow{\partial_{d}}\right),\left(\overrightarrow{\partial_{1}}, \ldots, \overrightarrow{\partial_{d}}\right)\right)\left(\exp \left(s_{i} X^{i}\right), \exp \left(t_{j} X^{j}\right)\right)
$$

where we have explicitly displayed the dependence of the bi-differential operator $\hat{\mathcal{D}}$ on the vector field $\mathbf{X}$, the structure constants $\mathbf{f}$ and the higher order derivatives.

In terms of the symbol $D$ this last expression is given by

$$
\exp \left(s_{i} X^{i}\right) \star \exp \left(t_{j} X^{j}\right)=D\left(\mathbf{X}, \mathbf{f},\left(s_{1}, s_{2}, \ldots, s_{d}\right),\left(t_{1}, t_{2}, \ldots t_{d}\right)\right) \exp \left(s_{i} X^{i}\right) \exp \left(t_{j} X^{j}\right)
$$

where $D$ is given by the Eq. (2.5). For future convenience we write another form for the star-product (2.9)

$$
\exp \left(s_{i} X^{i}\right) \star \exp \left(t_{j} X^{j}\right)=\exp \left(H\left(s_{i} X^{i}, t_{j} X^{j}\right)-s_{i} X^{i}-t_{j} X^{j}\right) \exp \left(s_{i} X^{i}\right) \exp \left(t_{j} X^{j}\right)
$$




$$
=\exp \left(H\left(s_{i} X^{i}, t_{j} X^{j}\right)\right)
$$

where it is explicitly shown the realization through the $\mathrm{CBH}$-formula. Or in general from Eq. (2.8) the star-product is given by

$$
\begin{gathered}
\exp \left(s_{i} X^{i}\right) \star \exp \left(t_{j} X^{j}\right) \\
=\exp \left(s_{i} X^{i}\right)\left(1+\sum_{n=1}^{\infty} \frac{1}{2^{n}} X^{k_{1}} f_{k_{1}}^{i_{1} j_{1}} \ldots X^{k_{n}} f_{k_{n}}^{i_{n} j_{n}} \overleftarrow{\partial}_{i_{1}} \ldots \overleftarrow{\partial}_{i_{n}} \cdot \vec{\partial}_{j_{1}} \ldots \vec{\partial}_{j_{n}}+\ldots\right) \exp \left(t_{j} X^{j}\right)
\end{gathered}
$$

where ... denotes the rest of the terms in the expansion of the exponential given in Eq. (2.6). For further details of the properties of the CBH-quantization see [15].

\subsection{Kontsevich's Quantization of $\mathcal{G}^{*}$}

Now we recall the basic formulas from the Kontsevich's construction of the starproduct on any Poisson manifold $M$ [11].

The Kontsevich's formula is a sum over a suitable class of oriented labeled admissible graphs $\mathbf{G}_{n}$ of order $n \geq 0$ (constructed from $n$ wedges graphs). An admissible graph $\Gamma$ is an element of $\mathbf{G}_{n}$ if it is constructed from $n+2$ aerial vertices $\{1,2, \ldots, n\}$ and two ground fixed vertices $\{L, R\}$ with $V_{\Gamma}=\{1,2, \ldots, n\} \sqcup\{L, R\}$ and $2 n$ edges from the set of edges of $\Gamma, E_{\Gamma}$ such that for each vertex $v_{k}$ of $\{1,2, \ldots, n\}$ there are a pair of edges emanating from $v_{k}$ to any other of the aerial vertices except for $v_{k}$ itself $i . e$. there are no loops. The pair of edges emanating from each $v_{k}$ of the aerial vertices are labeled as $e_{k}^{1}$ and $e_{k}^{2}$.

In [11] Kontsevich found a correspondence between each graph $\Gamma$ of $\mathbf{G}_{n}$ and bidifferential operators in the form:

$$
\Gamma \in \mathbf{G}_{n} \Leftrightarrow B_{\Gamma, \alpha}: C^{\infty}(M) \otimes C^{\infty}(M) \rightarrow C^{\infty}(M)
$$

with $M$ the relevant' Poisson manifold. Thus the Kontsevich star-product is

$$
f \star_{K} g \equiv \sum_{n=0}^{\infty} \hbar^{n} \sum_{\Gamma \in \mathbf{G}_{n}} W_{\Gamma} B_{\Gamma, \alpha}(f, g)
$$

with $W_{\Gamma}$ is given by 


$$
W_{\Gamma} \equiv \frac{1}{(2 \pi)^{n}} \int_{\mathcal{H}_{n}} \bigwedge_{i=1}^{n} d \phi_{e_{k}^{1}}^{h} \wedge d \phi_{e_{k}^{2}}^{h},
$$

where $\mathcal{H}_{n}$ is the space of configurations of $n$ numbered pairwise distinct points on $\mathcal{H}$ given by the $n$-punctured upper half-plane $\mathcal{H}=\{z \in \mathbb{C} / \operatorname{Im}(z)>0\}$ endowed with the Lobachevsky metric. The integral determining the weights (2.13) it was proved to be

absolutely convergent [11]. Finally $\phi_{e_{k}^{1}}^{h}$ and $\phi_{e_{k}^{2}}^{h}$ are harmonic functions on $\mathcal{H}_{n}$ representing the angles between $l(p, \infty)$ and the edges $e_{k}^{1}$ and $e_{k}^{2}$ respectively, measured counterclockwise from $l(p, \infty)$ and $B_{\Gamma, \alpha}(f, g)$ is given by

$$
\begin{aligned}
B_{\Gamma, \alpha}(f, g)= & \sum_{I: E_{\Gamma} \rightarrow\{1, \ldots, d\}}\left[\prod_{k=1}^{n}\left(\prod_{e \in E_{\Gamma}, e(*, k)} \partial_{I}(e)\right) \alpha^{\left.I\left(e_{k}^{1}\right) I\left(e_{k}^{2}\right)\right]}\right. \\
& \times\left(\prod_{e \in E_{\Gamma}, e(*, L)} \partial_{I}(e)\right) f \times\left(\prod_{e \in E_{\Gamma}, e(*, R)} \partial_{I}(e)\right) g .
\end{aligned}
$$

In the particular case when the Poisson manifold $M$ is identified with $\mathcal{G}^{*}$, the Kontsevich's formula has very interesting interconnections with the others deformation quantizations of $\mathcal{G}^{*}$. It was shown in Refs. [15, 16, 18] that taking the vector fields $X$ and $Y$ from Eq. (2.4) to be located at the ground vertices $L$ and $R$ of the Kontsevich's construction, the only possible graphs associated to the bi-differential operators $B_{\Gamma, \alpha}$, are of two types: $(i)$ Loop graphs and ( $i i)$ symmetric-admissible graphs. For the particular case of nilpotent Lie algebras, the loop graphs contribution is vanishing [15] and the Kontsevich's deformation quantization star-product coincides exactly with the $\mathrm{CBH}$ star-product Eq. (2.11). For more general Lie algebras (no necessarily nilpotent) loop graphs are non-zero but still is possible to find a mapping providing the equivalence between the Kontsevich's and CBH star-products [15, 16, 18].

\section{D-branes on Group Manifolds}

In this section we describe some elementary facts about D-branes in group manifolds which we will need in the next section. Another description of D-branes in group manifolds in the boundary state formalism would be relevant for the deformation quantization theory [5,6,6]. However for the purposes of this paper we will follows the lines of Refs. [4, 9]. 
It is well known that the propagation of closed strings in a group manifold $\mathbf{G}$ is described by the WZW Lagrangian for the field $g: \Sigma \rightarrow \mathbf{G}$ [3, 31] satisfy the closed string boundary conditions $g(0, \tau)=g(2 \pi, \tau)$

$$
L(g)=-\frac{k}{8 \pi} \int_{\Sigma} d^{2} \sigma \sqrt{h} h^{i j} \operatorname{Tr}\left(g^{-1} \partial_{i} g \cdot g^{-1} \partial^{j} g\right)-i k \Gamma(g),
$$

where $h^{i j}$ is a metric on the closed Riemann surface $\Sigma$, Tr is an invariant form on the Lie algebra $\mathcal{G}$ of $\mathbf{G}$ and $\Gamma(g)$ is the Wess-Zumino term which is given by

$$
\Gamma(g)=\int_{B} \widetilde{g}^{*} \chi=\frac{1}{12 \pi} \int_{B} d^{3} \sigma \varepsilon^{i j k} \operatorname{Tr}\left(g^{-1} \partial_{i} g \cdot g^{-1} \partial_{j} g \cdot g^{-1} \partial_{k} g\right) .
$$

Here $B$ is a three-manifold which has as boundary $\Sigma$, i.e. $\partial B=\Sigma$, and $\chi$ is the left and right invariant three form on the group manifold $\mathbf{G}$ given by

$$
\chi=\frac{1}{12 \pi} \operatorname{Tr}\left(g^{-1} d g \wedge g^{-1} d g \wedge g^{-1} d g\right) .
$$

In the Eq. (3.2) $\widetilde{g}$ is an extension of $g$ to the volume $B$ i.e. $\widetilde{g}: B \rightarrow \mathbf{G}$. Such an extension exists if $\pi_{2}(\mathbf{G})=0$. Finally the coupling constant $k$ is an integer-valued constant and it is an element of $H^{3}(\mathbf{G}, \mathbb{Z})$. Actually the three-form $\chi$ on $\mathbf{G}$ is an integer-valued cohomology class, i.e. an element of $H^{3}(\mathbf{G}, \mathbb{Z})$. This implies that $\chi$ is a closed form on $\mathbf{G}$ but $\chi$ never is the differential of a global two-form $\omega$ over $\mathbf{G}$. That means that it is not possible to define a gobal two-form $\omega$ over $\mathbf{G}$.

For the case of open strings the Lagrangian (3.1) is not well defined. The reason of this is as follows: the propagation of open strings in $\mathbf{G}$ generates an open world-sheet $W$ on which it can be coupled to a global two-form $\omega$ on $\mathbf{G}$. For the reasons mentioned previously such a global form cannot be defined on $\mathbf{G}$. Thus for open strings the first term of the rhs of the Lagrangian (3.1) is still well defined but the second one is not.

Now in order to consider a D-brane configuration in $\mathbf{G}$, that is, two parallel submanifolds $\mathbf{H}_{1}$ and $\mathbf{H}_{2}$ of $\mathbf{G}$ and open strings stretched between these submanifolds [4, 9]. One can associate locally the required two-form on each submanifold $\mathbf{H}_{i} i=1,2$ i.e. $C_{i}$ on $\mathbf{H}_{i}$ for $i=1,2$, respectively. These forms satisfy

$$
\left.\chi\right|_{\mathbf{H}_{i}}=d C_{i} .
$$

That means that the $C_{i}$ forms are chosen such that their exterior derivative coincides with the restriction of the three-form $\chi$ on each submanifold $\mathbf{H}_{i}$. 
Ambiguities of the WZ-term for this D-brane configuration is given by [4]

$$
\delta \Gamma(g)=\Gamma(g)-\int_{P_{1}} g^{*}\left(C_{1}\right)-\int_{P_{2}} g^{*}\left(C_{2}\right) .
$$

where $P_{i}$ is a two-dimensional submanifold of $\mathbf{H}_{i}$ for $i=1,2$ respectively and this is precisely the two-dimensional projection to $\mathbf{H}_{i}$ of the volume generated by the variation of the word-sheet $W$ connecting $\mathbf{H}_{1}$ and $\mathbf{H}_{2}$ in the group manifold $\mathbf{G}$.

Notice that this action is well defined only locally. Thus the fields $g$ are restricted to live in the corresponding submanifold $\mathbf{H}_{i}$ of $\mathbf{G}$. In general $\mathbf{H}_{i}$ are not necessary to be a subgroup of $\mathbf{G}$. However the case in which it coincides with the conjugacy classes of $\mathbf{G}$ is quite interesting and it will be discussed in the next section in the context of deformation quantization of adjoint and coadjoint orbits of $\mathbf{G}$.

Thus WZW theory for open strings with their end points on the D-branes is well defined in terms of the triple $\left(\chi, C_{1}, C_{2}\right) . m$ strings propagating in $\mathbf{G}$ and satisfying the Dirichlet boundary conditions on the group submanifolds $\mathbf{H}_{i}$ as above is given by

$$
L_{D}(g)=-\frac{k}{4 \pi} \int_{W} d^{2} \sigma \operatorname{Tr}\left(g^{-1} \partial_{z} g \cdot g^{-1} \partial_{\bar{z}} g\right)+\frac{k}{12 \pi i} \int_{B} \widetilde{g}^{*} \chi-\left.\frac{k}{4 \pi} \sum_{m} \int_{W_{m}} \widetilde{g}^{*}\right|_{W_{m}} C_{m}
$$

where $z$ and $z$ are the complex coordinates on the disc $W$. The sum in Eq. (3.6) is over the number of components of the boundary on the disc $W$. Action (3.6) is well defined and it is completely specified by the forms $\left(\chi, C_{m}\right)$. Such a pair can be interpreted as an element of the relative cohomology chain $H^{*}\left(\mathbf{G} / \mathbf{H}_{m}, \mathbb{Z}\right)$. The world-sheet $W$ and in general higher-dimensional world-volumes $W_{k}$ of dimension $k$ can be interpreted as $k$-dimensional cycles of the relative homology chain $H_{*}\left(\mathbf{G} / \mathbf{H}_{m}, \mathbb{Z}\right)$ i.e. $k$-dimensional submanifolds of $\mathbf{G}$ whose boundary lies in the $\mathbf{H}_{m}$ D-branes. Thus the relative integer homology classifies the different D-branes in the group manifold $\mathbf{G}$. The $\mathbb{Z}$-valued (co)homology is necessary in order that the Feynman path integral be well defined.

Thus the WZW action for an open string in the D-brane configuration is given by

$$
L_{W}(g)=-\frac{k}{4 \pi} \int_{W} d^{2} \sigma \operatorname{Tr}\left(g^{-1} \partial_{z} g \cdot g^{-1} \partial_{\bar{z}} g\right)-\frac{k}{4 \pi} \int_{W} B
$$

where $B=\widetilde{g}(G)$ with $G$ is the local two-form defined on the image of $W$ under $g$, such that $\chi=d G$. 
If the boundary of $W$ is empty then the term $\int_{W} B$ is invariant under the transformation $B \rightarrow B+d \Lambda$. But if the case is such that the boundary of $W$ is no empty then the term $\int_{W} B$ will transforms as

$$
\int_{W} B \rightarrow \int_{W} B+\int_{\partial W \cap \mathbf{H}} \Lambda
$$

At this step one can imitate the standard case of D-branes in type II string theories [32] by noting that $C-B$ on the D-brane is a closed form, then locally we have abelian degrees of freedom $A$ in the boundaries of the open string attached to the D-brane and it is given by

$$
\int_{\partial W \cap \mathbf{H}} A
$$

Thus the gauge invariance can be restored if the gauge transformation for the $B$-field is accompanied by the transformation of $A$ in the form $A \rightarrow A-\left.\Lambda\right|_{\mathbf{H}}$. The gauge invariant field strength is not $F=d A$ but the combination $\mathcal{B}=F-B$. Thus the effective action is

$$
L_{W}(g)=-\frac{k}{4 \pi} \int_{W} d^{2} \sigma \operatorname{Tr}\left(g^{-1} \partial_{z} g \cdot g^{-1} \partial_{\bar{z}} g\right)-\frac{k}{4 \pi} \int_{W} \mathcal{B} .
$$

The gauge field in two-dimensions has no-propagating degrees of freedom and it implies that field strength $\mathcal{B}$ should be constant, $\mathcal{B}=\frac{1}{2} \varepsilon^{i j} \mathcal{B}_{i j}$. Thus a $\mathcal{B}$-field term can be consistly introduced into the WZW description of open strings in group manifolds when D-brane configurations are included. In the next section we will show that precisely this $\mathcal{B}$-field term will plays a crucial role in the derivation of the Kontsevich's star-product for the dual Lie algebra $\mathcal{G}^{*}$.

In Ref. [8] it was shown that the explicit form of $\mathcal{B}$ can also be extracted from the boundary condition in the closed string picture $J=-\bar{J}$ where $J$ and $\bar{J}$ are the holomorphic and anti-holomorphic chiral currents of the WZW model [5,6, 7,8] and it is given by

$$
-\frac{k}{4 \pi} \int_{W} \varepsilon^{i j} \operatorname{Tr}\left(g^{-1} \partial_{i} g \cdot \frac{1+A d(g)}{1-A d(g)} \cdot g^{-1} \partial_{j} g\right)
$$

where $A d(g)$ is the adjoint action of $\mathbf{G}$ on the Lie algebra $\mathcal{G}$. It is given by $\operatorname{Ad}(g) X:=$ $\left.\frac{d}{d t}\right|_{t=0} g(\operatorname{expt} X) g^{-1}$ for all $g$ in $\mathbf{G}$. In this picture, the D-brane is identified with the (co)adjoint orbit (conjugacy class) of $\mathbf{G}$. This result leads to a reconciliation between the approach 四 and the boundary state description [0, 6 ]. 


\section{D-branes in G and the Kontsevich Star Product on $\mathcal{G}^{*}$}

The aim of this section is the derivation of the Kontsevich's formula of deformation quantization for the dual of a Lie algebra $\mathcal{G}^{*}$. We will argue that this formula is encoded in the theory of D-branes on group manifolds as given in Refs. [4, 8,9] and reviewed in the previous section.

First of all we shall recall that the effective WZW field theory on the D-brane in a group manifold $\mathbf{G}$ is given by the Eq. (3.10). For definiteness we will take a compact and simple group manifold $\mathbf{G}$ whose dual Lie algebra $\mathcal{G}^{*}$ be provided with the Kirillov-Poisson structure. It is an easy matter to show that Eq. (3.10) can be rewritten as

$$
L_{W}(g)=-\frac{k}{4 \pi} \int_{W} d^{2} \sigma \operatorname{Tr}\left(g^{-1} \partial_{z} g \cdot g^{-1} \partial_{\bar{z}} g\right)-\frac{1}{\pi k} \int_{W} d^{2} \sigma B_{a b} J^{a}(z) \bar{J}^{b}(\bar{z}),
$$

where $B_{a b}$ is an antisymmetric and constant two-form on $\mathbf{H}$ and $J^{a}(z)$ and $\bar{J}^{b}(\bar{z})$ are the chiral currents of the WZW model and they are vector fields in $\mathcal{G}$ satisfying

$$
\begin{aligned}
& J(z)=J^{a} T_{a}=-\frac{1}{2} k g^{-1} \partial_{z} g, \\
& \bar{J}(\bar{z})=\bar{J}^{a} T_{a}=-\frac{1}{2} k g^{-1} \partial_{\bar{z}} g,
\end{aligned}
$$

where $\left\{T_{a}\right\}$ with $a=1, \ldots, \operatorname{dim}(\mathbf{G})$ is a basis of $\mathcal{G}$. It is well known from the theory of open strings that the disc $W$ can be transformed into the upper half-plane $\mathcal{H}$ with the boundary of the disc mapped into the boundary of $\mathcal{H}$ located at $\operatorname{Im}(z)=0$.

We consider the correlation functions on the disc $W$ of the operators $g$ located in 0 and 1 in the boundary of $W$. These correlation functions will be defined taking only the first term of the Lagrangian (4.1) in the Feynman path integral

$$
\langle g(1) \cdot g(0)\rangle=\frac{1}{Z} \int \mathcal{D} g g(1) \cdot g(0) \exp \left(\frac{k}{4 \pi} \int_{W} d^{2} \sigma \operatorname{Tr}\left(g^{-1} \partial_{z} g \cdot g^{-1} \partial_{\bar{z}} g\right)\right),
$$

where

$$
Z=\int \mathcal{D} g \exp \left(+\frac{k}{4 \pi} \int_{W} d^{2} \sigma \operatorname{Tr}\left(g^{-1} \partial_{z} g \cdot g^{-1} \partial_{\bar{z}} g\right)\right)
$$


Since the fields $g$ are $\mathbf{G}$-valued mappings it is natural to assume that the insertion operators in 0 and 1 on the boundary of the disc are given by

$$
g(1) \equiv \exp (J), \quad g(0) \equiv \exp (\bar{J})
$$

with

$$
J(z) \equiv J^{a}(z) T_{a}, \quad \bar{J}(\bar{z})=\bar{J}^{a}(\bar{z}) T_{a}
$$

where $J^{a}(z)$ and $\bar{J}^{a}(\bar{z})$ are the holomorphic and anti-holomorphic currents given in Eqs. (4.2) and (4.3).

We propose that the two-point correlation function for the complete action $L_{W}$ of Eq. (4.1) leads to the Kontsevich's formula of the deformation quantization of the dual Lie algebra $\mathcal{G}^{*}$ of the group manifold $\mathbf{G}$. This implies that the mentioned Kontsevich's formula is encoded in the theory of open strings with Dirichlet boundary conditions in group manifolds. Thus we should consider the complete action $L_{W}$ including the $B$-field term too. Coefficient of the $B$-field term goes as $1 / k$ and in the large- $k$ limit one can consider this term as a 'perturbation' and use the usual $1 / k$-perturbation theory. Before of proceeding with the $1 / k$-expansion we consider generically the effect of the $B$-field term in the two-point correlation functions. Thus one can define new (deformed) correlation functions $\langle g(1) \cdot g(0)\rangle^{B}$ for this 'perturbed' system in the form

$$
\langle g(1) \cdot g(0)\rangle^{B}=\frac{1}{Z}\left\langle g(1) \cdot g(0) \exp \left(+\frac{1}{\pi k} \int_{\mathcal{H}} d^{2} \sigma B_{a b} J^{a}(z) \bar{J}^{b}(\bar{z})\right)\right\rangle
$$

where now

$$
Z=\int \mathcal{D} g \exp \left(i L_{W}\right)
$$

In Eq. (4.8) we have replaced $W$ by $\mathcal{H}$. The point $(z, \bar{z})$ where the holomorphic $J(z)$ and anti-holomorphic $\bar{J}(\bar{z})$ chiral currents are evaluated, is a specific point of the interior of the $\mathcal{H}$-plane.

The product $g(1) \cdot g(0)$ inside the above correlation function is the usual group product

$$
g(1) \cdot g(0)=\exp (J) \cdot \exp (\bar{J})
$$

We can now apply the CBH-formula (2.1) to the above equation and it results 


$$
\exp (J) \cdot \exp (\bar{J})=\exp (H(J, \bar{J}))
$$

where

$H(J, \bar{J})=J^{a}(z) T_{a}+\bar{J}^{a}(\bar{z}) T_{a}+\frac{1}{2} T_{c} f_{a b}^{c} J^{a} \bar{J}^{b}+\frac{1}{12}\left(T_{e} f_{a d}^{e} f_{b c}^{d} J^{a} J^{b} \bar{J}^{c}+T_{e} f_{d c}^{e} f_{a b}^{d} J^{a} \bar{J}^{b} \bar{J}^{c}\right)+\ldots$

Here $\left\{T_{a}\right\} a=1, \ldots, \operatorname{dim}(\mathbf{G})$ is a basis of $\mathcal{G}$ and $f_{b c}^{a}$ are the structure constants of the Lie algebra of $\mathbf{G}$. At this level one can consider the set of generators $T_{a}$ of the Lie algebra $\mathcal{G}$ as local coordinate functions on $\mathcal{G}^{*}$ according to the correspondence $(2.3)$. Thus $\exp \left(J^{a} T_{a}\right)$ and $\exp \left(\bar{J}^{a} T_{a}\right)$ can be seen as functions on $\mathcal{G}^{*}$ i.e. as elements of $C^{\infty}\left(\mathcal{G}^{*}\right)$. It is very easy to remove the part $J+\bar{J}$ from the CBH-formula (4.12) and we get

$$
g(1) \cdot g(0)=D \hat{g}(1) \hat{g}(0)
$$

where $\hat{g}(1)=\exp \left(J^{a}(z) T_{a}\right)$ and $\hat{g}(0)=\exp \left(\bar{J}^{a}(\bar{z}) T_{a}\right)$ are seen as functions on $\mathcal{G}^{*}$ and $D$ is the symbol given by

$$
D=\exp \left(\frac{1}{2} T_{c} f_{a b}^{c} J^{a} \bar{J}^{b}+\frac{1}{12}\left(T_{e} f_{a d}^{e} f_{b c}^{d} J^{a} J^{b} \bar{J}^{c}+T_{e} f_{d c}^{e} f_{a b}^{d} J^{a} \bar{J}^{b} \bar{J}^{c}\right)+\ldots\right)
$$

We can substitute this last expression into the deformed correlation function (4.8) and it yields

$$
\langle g(1) \cdot g(0)\rangle^{B}=\frac{1}{Z}\left\langle D \hat{g}(1) \hat{g}(0) \exp \left(\frac{1}{\pi k} \int_{\mathcal{H}} d^{2} \sigma B_{a b} J^{a}(z) \bar{J}^{b}(\bar{z})\right)\right\rangle .
$$

In the large- $k$ limit one can perform the perturbative $1 / k$-expansion of the $B$-field term where we shall recall that $B_{a b}$ must be constant by the arguments outlined at the end of the Section 3. Thus we get

$\langle g(1) \cdot g(0)\rangle^{B}=\frac{1}{Z} \sum_{n=0}^{\infty}\left(+\frac{1}{\pi k}\right)^{n} \frac{1}{n !} \int_{\mathcal{H}_{n}} d z_{1} d \bar{z}_{1} \ldots d z_{n} d \bar{z}_{n}\left\langle D_{n} \hat{g}(1) \hat{g}(0) \prod_{j=1}^{n} B_{a_{j} b_{j}} J^{a_{j}}(z) \bar{J}^{b_{j}}(\bar{z})\right\rangle$

where $\mathcal{H}_{n}=\left\{\left(z_{1}, \ldots, z_{n}\right) / z_{i} \in \mathcal{H}, \operatorname{Im}\left(z_{j}\right)>\epsilon,\left|z_{j}-z_{i}\right|>\epsilon, i \neq j\right\}$ is a $2 n$-dimensional real manifold, $D_{n}$ is given by $D$ from Eq. (4.14) evaluated in the $n$-th coordinate pair $\left(z_{n}, \bar{z}_{n}\right)$. 
The fact that $\hat{g}(0)$ and $\hat{g}(1)$ can be seen as smooth functions on $\mathcal{G}^{*}$ it does not implies that they can be seen as functions on the disc $W$. Actually they are operators and one has to compute the remaining correlation functions on the disc. In order to do that one must give the operator product expansion (OPE) of the operators $g$ with the currents $J$ and $\bar{J}$. They are given by 3

$$
J^{a}(z) g(\xi)=-\frac{1}{z} T^{a} g(\xi)+\ldots
$$

and

$$
\bar{J}^{a}(\bar{z}) g(\xi)=\frac{1}{\bar{z}} g(\xi) T^{a}+\ldots
$$

where ... denotes the non-singular terms of the OPE. Applying these equations one can shown that the Eq. (4.16) turns out to be

$$
\begin{aligned}
\langle g(1) \cdot g(0)\rangle^{B}=\frac{1}{Z} \sum_{n=0}^{\infty}\left(-\frac{1}{\pi k}\right)^{n} \frac{1}{n !} \int_{\mathcal{H}_{n}} d z_{1} d \bar{z}_{1} \ldots d z_{n} d \bar{z}_{n} \prod_{k=1}^{n} \frac{1}{z_{k} \bar{z}_{k}} \\
\times\left\langle B_{a_{1} b_{1}} \ldots B_{a_{n} b_{n}} T^{a_{1}} T^{b_{1}} \ldots T^{a_{n}} T^{b_{n}} D_{n} \hat{g}(1) \hat{g}(0)\right\rangle .
\end{aligned}
$$

In terms of the bi-differential operator $\hat{\mathcal{D}}$ from Eq. (2.6) we get

$$
\langle g(1) \cdot g(0)\rangle^{B}=\sum_{n=0}^{\infty}\left(-\frac{1}{\pi k}\right)^{n} \frac{1}{n !} \int_{\mathcal{H}_{n}} d z_{1} d \bar{z}_{1} \ldots d z_{n} d \bar{z}_{n} \prod_{k=1}^{n} \frac{1}{z_{k} \bar{z}_{k}}\left\langle\hat{\mathcal{D}}_{n}(\hat{g}(1), \hat{g}(0))\right\rangle
$$

where we have absorbed the terms with $B$ 's and $T$ 's in the normalization factor. Thus the above formula can be written as

$$
\langle g(1) \cdot g(0)\rangle^{B}=\sum_{n=0}^{\infty}\left(-\frac{1}{\pi k}\right)^{n} \sum_{\Gamma} W_{\Gamma}\left\langle\hat{\mathcal{D}}_{\Gamma}(\hat{g}(1), \hat{g}(0))\right\rangle
$$

where

$$
W_{\Gamma}=\frac{1}{n !} \int_{\mathcal{H}_{n}} d z_{1} d \bar{z}_{1} \ldots d z_{n} d \bar{z}_{n} \prod_{k=1}^{n} \frac{1}{z_{k} \bar{z}_{k}}
$$

with $\Gamma$ representing an admissible graph constructed from vertices and edges following the recipe of subsection 2.3 .

Thus one can see that the correlation function $\langle g(1) \cdot g(0)\rangle^{B}$ looks like the Kontsevich's product for the Lie algebra $\mathcal{G}^{*}$ which is given by 


$$
\hat{g}(1) \star_{K} \hat{g}(0) \equiv\langle g(1) \cdot g(0)\rangle^{B}=\sum_{n=0}^{\infty}\left(-\frac{1}{\pi k}\right)^{n} \sum_{\Gamma \in \mathbf{G}_{n}} W_{\Gamma} B_{\Gamma}(\hat{g}(1), \hat{g}(0))
$$

where we have identified $\hat{\mathcal{D}}_{\Gamma}(\hat{g}(1), \hat{g}(0))$ to $B_{\Gamma}(\hat{g}(1), \hat{g}(0))$ just as one would expect.

Formula (4.23) reproduces exactly the Konsevich's star-product Eq. (2.12) defined on the space of smooth functions on the dual Lie algebra $\mathcal{G}^{*}$ of the group manifold $\mathbf{G}$. It is remarkable that the Kontsevich's formula was obtained explicitly as a $1 / k$-expansion for the large- $k$ limit. This is a typical characteristic of a string perturbative expansion in its string coupling constant in the correspondence between the topological sigma model for open strings and the Chern-Simons effective field theory [33]. In fact, the close relation between Chern-Simons and WZW model leads us to think that a description of the Kontsevich's formula in terms of the Feynman diagrams of the Chern-Simons theory is possible. Actually it is no surprising form the results of [22, 23, 25].

Several comments are in order. The first one concerns with the relation with the boundary states description of D-branes in group manifolds [5, 6, [7]. From there it is known that Dirichlet boundary conditions for open strings in groups can be encoded in the algebraic structure of the corresponding superconformal algebra. Within this prescription it was shown in [8] that for D-branes $\mathbf{H}$ with group structure, i.e. subgroups of $\mathbf{G}$, for instance adjoint or coadjoint orbits $\mathbf{C}$ of $\mathbf{G}$. Boundary state prescription implies that open strings can end on this orbits $\mathbf{C}$ defining D-particles. From [6] if $\mathbf{C}$ constitutes a Lagrangian submanifold of $\mathbf{G}$ then $\left.T \mathbf{G}\right|_{\mathbf{C}}=T \mathbf{C} \oplus N \mathbf{C}$, where $T \mathbf{C}$ and $N \mathbf{C}$ are the respective tangent and normal bundle to $\mathbf{C}$. If we have a distinguished point (the identity) it turns out to be $\mathcal{G}=\mathcal{C} \oplus \mathcal{C}^{\perp}$ at the level of Lie algebras. Now if we assume that the boundary components of the disc are mapped into the real part of $\mathcal{G}$ i.e. are mapped to $\mathcal{C}$, then the fields of the Lagrangian (4.1) will be $\mathcal{C}$-valued fields and therefore the deformation quantization formula Eq. (4.23) will describe the deformation quantization of the coadjoint orbits $\mathbf{C}$. In particular, for semi-simple Lie groups $\mathbf{G}$, the corresponding star-product fom the $\mathrm{CBH}$-quantization should gives the quantization described in [34].

Following Ref. [35] it is well known that a topological field theory should lives in the corresponding D-brane wrapped in the Lagrangian submanifolds of $\mathbf{G}$. This topological field theory is provided by the effective field theory on the D-brane $W$ given by the WZW Lagrangian for open strings or its extensions to gauged WZW-theory [6, 36]. It is expected that a deeper treatment of the BRST and BV approach of WZW theory of D-branes would be a better framework to understand the Kontsevich's formula for $\mathcal{G}^{*}$ and its description 
in terms of the formality conjecture in a similar way than Cattaneo and Felder [22] found the Kontsevich's formula from a topological non-linear sigma model for open strings by using the BV quantization method. We leave this subject for a future investigation.

\section{Concluding Remarks}

The close relation between deformation quantization theory (and in general noncommutative geometry) and the theory of D-branes has appeared in diverse ways in the literature [22, 23, 21, 25, 27]. In the present paper we found new evidence which favored this relation. Here we have explored some interplay between the D-brane theory on the group manifold $\mathbf{G}$ and the Kontsevich's star-product on the dual Lie algebra $\mathbf{G}^{*}$ of the group $\mathbf{G}$. Our derivation has some similarity to the derivation of the Kontsevich's product on $\mathbb{R}^{n}$ by using the two-point correlation functions from the perturbative theory of open strings in $\mathbb{R}^{n}$ in a background $B$-field [25]. However various different things are in order. In our case the relevant theory is the effective WZW-field theory describing D-branes living in the group manifold $\mathbf{G}$ [4]. This effective field theory is given by the pairing in relative (co)homology chain $H(\mathbf{G} / \mathbf{H}, \mathbb{Z})$. This implies the consistent existence of a background $B$ field term which is coupled to the world-sheet $W$. This $B$-field term, as we have showed, it generates a $1 / k$-expansion which can be (under some specifications) identified with the Kontsevich's formula for $\mathcal{G}^{*}$.

It is interesting to conclude from our results that the Kontsevich's formula on $\mathcal{G}^{*}$ given Eq. (4.23) can be seen as a $1 / k$-perturbative expansion of the WZW effective field theory on a D-brane in G. Moreover Witten has showed in [33] that Chern-Simons perturbation theory can been seen as a perturbative open string theory with the $1 / k$-expansion of the Chern-Simons theory coinciding with the perturbative expansion of the string theory. Chern-Simons field theory represents the low energy effective field theory of the string theory with the string coupling constant given by $1 / k$. Thus our result of getting the Kontsevich's formula as a perturbative expansion in the string coupling constant confirms the current statement that the deformation quantization of any Poisson manifold needs from stringly concepts [22,25,21].

It is very well known that $1 / k$-perturbative expansions in WZW theory are related to $1 / k$-perturbative expansions in the Chern-Simons theory. Thus one can, in principle, 
to translate our results in terms of a topological perturbative invariants of Chern-Simons theory. It would be interesting to study the Kontsevich's formula as given in [22] and to look for its relation to the Rozansky-Witten invariants [37]. It would be interesting to explore also further interplay between the Poisson-Lie T-duality of the WZW theory for D-branes [4] and the relevant algebraic relations involved in the quantization of Lie bialgebras as worked out in [19]. Also, following [25] one would trying to generalize the CBHquantization and its relation to Kontsevich's formula to curved manifolds (Riemannian phase spaces) through the application of the boundary deformation theory [38]. It would be interesting to compare these results to those obtained by Bordemann et al [39] in the context of Fedosov's formalism.

Finally, the search for a deeper interplay between the string theory tools as: conformal field, theory, Maldacena's conjecture, D-branes and D-branes in group manifolds, moduli spaces etc. and mathematics tools as: formality conjecture, operads, motives etc. pointed out in [21] deserves intensive further investigation.

\section{Acknowledgements}

One of us (H. G.-C.) would like to thank Prof. E. Witten for his hospitality at the Institute for Advanced Study. It is a pleasure to thank I. Carrillo for useful discussions. 


\section{References}

[1] J. Polchinski, "TASI Lectures on D-branes", in Fields Strings and Duality TASI-96, Eds. C. Efthimiou and B. Greene, World Scientific (1997), hep-th/9611050.

[2] M. Douglas, "Superstring Dualities, Dirichlet Branes and the Small Scale Structure of Space", hep-th/9610041.

[3] D. Gepner and E. Witten, "String Theory on Group Manifolds", Nucl. Phys. B 278 (1986) 493-549.

[4] C. Klimčik and P. Ševera, "Poisson-Lie T-duality: Strings and D-branes", Phys. Lett. B 376 (1996) 82; "Open Strings and D-branes in WZNW Models", Nucl. Phys. B 488 (1997) 653-676.

[5] M. Kato and T. Okada, "D-branes on Group Manifolds", Nucl. Phys. B 499 (1997) 583, hep-th/9612148.

[6] S. Stanciu. "D-branes in Kazama-Susuki Models", Nucl. Phys. B 526 (1998) 295, hep-th/9708166.

[7] A. Recknagel and V. Schomerus, "D-branes in Gepner Models", Nucl. Phys. B 531 (1998) 185, hep-th/9712186.

[8] A. Yu. Alekseev and V. Schomerus, "D-branes in the WZW Model", hep-th/9812193.

[9] K. Gawedzki, "Conformal Field Theory: A Case Study", hep-th/9904145.

[10] F. Bayen, M. Flato, C. Fronsdal, A. Lichnerowicz and D. Sternheimer, "Deformation Theory and Quantization I;II", Ann. Phys. 111 (1978) 61;111; M. De Wilde and P.B.A. Lecomte, Lett. Math. Phys. 7 (1983) 487; H. Omory, Y. Maeda and A. Yoshioki, "Weyl Manifolds and Deformation Quantization", Adv. Math. 85 (1991) 224; B. Fedosov, "A Simple Geometrical Construction of Deformation Quantization", J. Diff. Geom. 40 (1994) 213.

[11] M. Kontsevich, "Deformation Quantization of Poisson Manifolds I", q-alg/9709040.

[12] S. Barannikov and M. Kontsevich, "Frobenius Manifolds and Formality of Lie Algebras of Polyvector Fields", alg-geom/9710032.

[13] S. Gutt, "An Explicit *-product on the Cotangent Bundle of a Lie Group", Lett. Math. Phys. 7 (1983) 249-258.

[14] S. Asin Lares, "On Tangential Properties of the Gutt *-Product", J. Geom. Phys. 24 (1998) 164.

[15] V. Kathotia, "Kontsevich's Universal Formula For Deformation Quantization and the Campbell-Baker-Hausdorff Formula, I", math.QA/9811174.

[16] G. Dito, "Kontsevich Star-product on the Dual of a Lie Algebra", math.QA/9905080.

[17] B. Mielnik and J.F. Plebański, "Combinatorial approach to Baker-Campbell-Hausdorff Exponents" Ann. Inst. Henri Poincaré 12 (1970) 215-254.

[18] B. Shoikhet, "On the Kontsevich and the CBH Deformation Quantization of a Linear Poisson Structure", math.QA/9903036. 
[19] P. Etingof and D. Kazhdan, "Quantization of Lie bialgebras, I;II;III", Sel. math., new ser. 2 (1996) 1; 4 (1998) 213; 4 (1998) 233.

[20] D. Tamarkin, "Another Proof of M. Kontsevich Formality Theorem", math.QA/9803025.

[21] M. Kontsevich, "Operands and Motives in Deformation Quantization", math.QA/9904055.

[22] A.S. Cattaneo and G. Felder, "A Path Integral Approach to the Kontsevich Quantization Formula", math.QA/9902090.

[23] A. Schwarz, "Quantum Observables, Lie Algebra Homology and TQFT", hepth/9904168.

[24] I.Ya. Aref'eva and I.V. Volovich, "Noncommutative Gauge Fields on Poisson Manifolds", hep-th/9907114.

[25] V. Schomerus, "D-branes and Deformation Quantization", JHEP 06 ((1999) 030, hepth/9903205.

[26] A. Connes, M.R. Douglas and A. Schwarz, "Noncommutative Geometry and Matrix Theory: Compactification on Tori", JHEP 02 (1998) 003, hep-th/9711162.

[27] M. Douglas, "Two Lectures on D-Geometry and Noncommutative Geometry", hepth/9901146.

[28] F. Ardalan, H. Arfaei and M.M. Sheikh-Jabbari, "Dirac Quantization of Open Strings and Noncommutativity in Branes", hep-th/9906161.

[29] Chong-Sun Chu and Pei-Ming Ho, "Constrained Quantization of Open Strings in Background B-field and Noncommutative D-brane", hep-th/9906192.

[30] H. García-Compeán, "On the Deformation Quantization Description of Matrix Compactification", Nucl. Phys. B 541 (1999) 651-670.

[31] E. Witten, "Nonabelian Bosonization in Two Dimensions", Commun. Math. Phys. 92 (1984) 455-472.

[32] E. Witten, "Bound States of Strings and p-Branes", Nucl. Phys. B 460 (1996) 335, hep-th/9510135.

[33] E. Witten, "Chern-Simons Gauge Theory as a String Theory", hep-th/9207094.

[34] R. Fioresi and M.A. Lledó. "On the Deformation Quantization of Coadjoint Orbits of Semisimple Groups", math.Q.A./9906104.

[35] M. Bershadsky, C. Vafa and V. Sadov, "D-branes and Topological QFT's", Nucl. Phys. B 463 (1996) 420.

[36] E. Witten, "The $N$-matrix Model and Gauged WZW Models, Nucl. Phys. B 371 (1992) 191.

[37] L. Rozansky and E. Witten, "Hyper-Kähler Geometry and Invariants for Threemanifolds", Sel. math., new ser. 3 (1997) 401.

[38] A. Recknagel and V. Schomerus, "Boundaries Deformation Theory and Moduli Spaces of D-branes", Nucl. Phys. B 545 (1999) 233, hep-th/9811237.

[39] M. Bordemann, N. Neumaier, S. Waldmann, Commun. Math. Phys. 198 (1998) 363. 